\title{
Modeling of Liquid Film Flow during Spin-Coating; Marangoni-Bénard Instability in Parallel Basic Flow
}

\author{
Kohei Ono $^{1}$, Suguru Shiratori ${ }^{1}$, Kenjiro Shimano ${ }^{1}$, Hideaki Nagano ${ }^{1}$ \\ ${ }^{1}$ Dept. Mechanical Systems Engineering, Tokyo City University \\ 1-28-1 Tamazutsumi, Setagaya-ku, Tokyo, Japan \\ g2081011@tcu.ac.jp (K. Ono); sshrator@tcu.ac.jp (S. Shiratori)
}

\section{Extended Abstract}

\section{Scope}

This study includes the contents of instability of the flow and mass and heat transfer in microstructure-manufacturing. By reproducing striation numerically that appears during spin-coating contributes to the development of industry.

\section{Objectives}

Radial spoke-like thickness variations called striation may appear in spin-coated films, which are used in the industrial products, for instance, semiconductor and MEMS (Micro Electro-Mechanical Systems) devices. The striation lowers the final quality of the product and is strongly required to be avoided or suppressed.

A hypothesis of striation generation process has been suggested that the convection cell of widely known MarangoniBénard instability is stretched by the background flow [1]. The experimental research [2] suggested that the practical striation which involves rather complicated structures was generated through multi-step transitions of the flow. In order to discuss further on the multi-step transitions, information on the detailed flow field is necessary, however, it cannot be obtained from this experiment [2], because only the thickness variations were measured.

In the present study, we executed 3-dimensional time-dependent numerical simulation of the flow during spin-coating in order to clarify how the striation is generated. We investigated whether the parallel basic flow became unstable under the certain strength of Marangoni effect.

\section{Results}

We obtained the spoke-like spatial variations of the flow and concentration fields numerically and compared these results with thickness variations obtained by the experiments [2]. One of the main features in the experimentally obtained striations are qualitatively reproduced by the present numerical simulation. The spoke-like spatial variations are generated in the numerically obtained concentration field and the spokes are fragmented when the body force is small. In the present study, the constant parallel body force was applied for the background flow, although the body force acting on the practical striations is centrifugal force of which intensity depends on the radial position. From the fact that the spoke-like spatial variations in concentration field is obtained by the constant body force model, we can conclude that the constant body force is enough to reproduce the essence of the spoke-like spatial variations in the striation.

In the present study, although we succeeded in reproducing the spoke-like spatial variations, multi-step transitions which are another main feature have not been reproduced yet. The reproduction of this feature will be the subject of further studies.

\section{References}

[1] D.E. Haas and D.P. Birnie. "Evaluation of thermocapillary driving forces and in the development of striations during and the spin coating process", J. Materials Sci., 2002.

[2] S.Shiratori, D.Kato, K.Sugasawa, H.Nagano, K.Shimano "Spatio-temporal thickness variation and transient Marangoni number in striations during spin coating”, Int. J. Heat Mass Trans., vol.154 119678, 2020. 